This item was submitted to Loughborough's Research Repository by the author.

Items in Figshare are protected by copyright, with all rights reserved, unless otherwise indicated.

\title{
Voltage-dependent quantum efficiency measurements of amorphous silicon multijunction mini-modules
}

PLEASE CITE THE PUBLISHED VERSION

http://dx.doi.org/10.1016/j.solmat.2010.03.039

PUBLISHER

(C) Elsevier B.V.

VERSION

AM (Accepted Manuscript)

LICENCE

CC BY-NC-ND 4.0

\section{REPOSITORY RECORD}

Hibberd, Christopher J., Foteini Plyta, Christos Monokroussos, Martin Bliss, Thomas R. Betts, and Ralph Gottschalg. 2019. "Voltage-dependent Quantum Efficiency Measurements of Amorphous Silicon Multijunction Mini-modules". figshare. https://hdl.handle.net/2134/6703. 
This item was submitted to Loughborough's Institutional Repository (https://dspace.lboro.ac.uk/) by the author and is made available under the following Creative Commons Licence conditions.

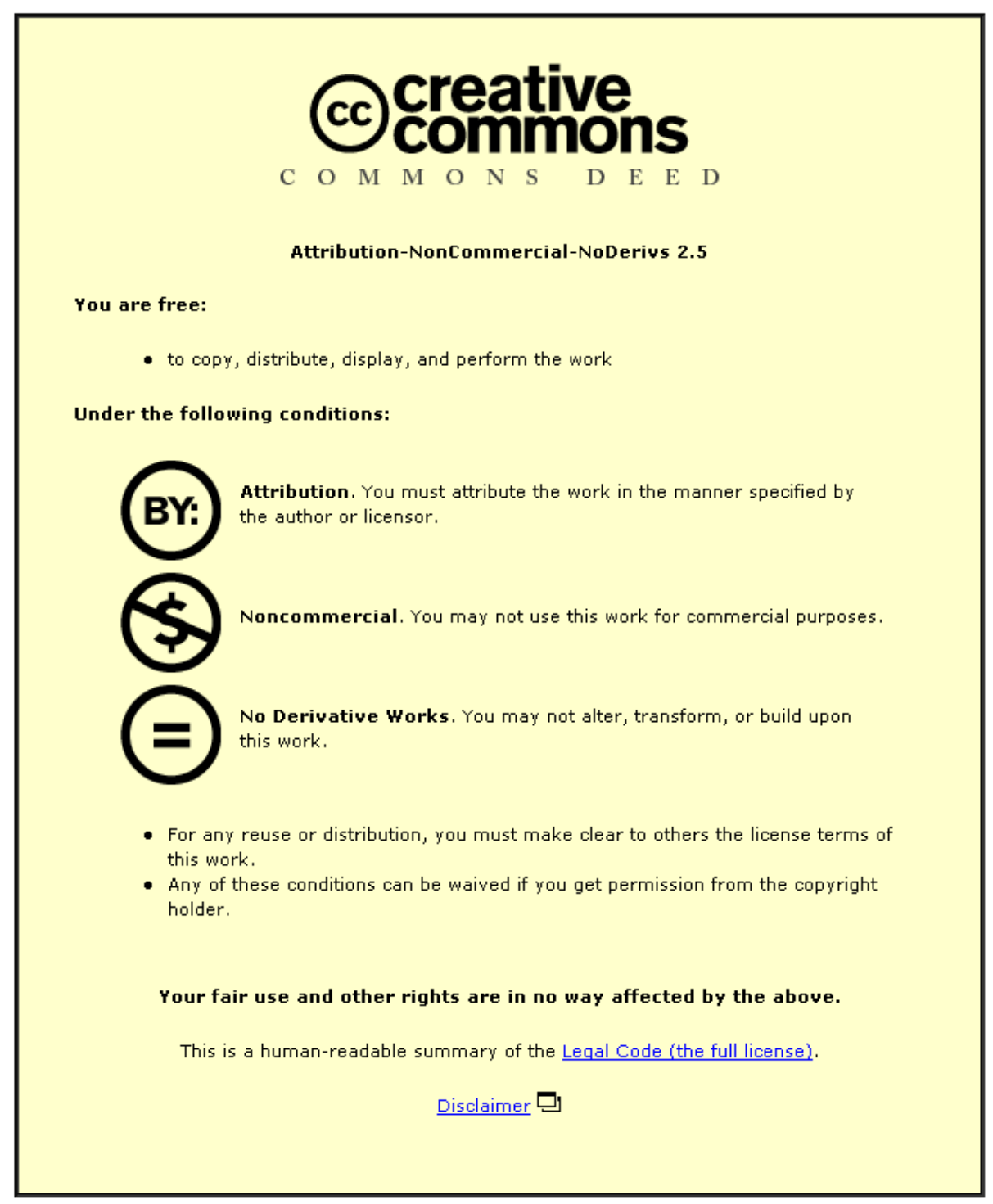

For the full text of this licence, please go to: http://creativecommons.org/licenses/by-nc-nd/2.5/ 


\title{
Voltage-dependent quantum efficiency measurements of amorphous silicon multi- junction mini-modules
}

\author{
C. J. Hibberd, F. Plyta, C. Monokroussos, M. Bliss, T.R. Betts and R. Gottschalg*
}

Centre for Renewable Energy Systems Technology (CREST), Holywell Park GX Area, Department for Electronic and Electrical Engineering, Loughborough University, Leicestershire, LE11 3TU, UK

*Corresponding author: R.Gottschalg@lboro.ac.uk

\begin{abstract}
Multi-junction solar cells have the potential to provide higher efficiencies than single junction devices and to reduce the impact of StaeblerWronski degradation on amorphous silicon (a-Si) devices. They could, therefore, reduce the cost of solar electricity. However, their characterization presents additional challenges over that of single junction devices. Achieving acceptable accuracy of any current-voltage calibration requires correction of the current-voltage data with external quantum efficiency measurements and spectral mismatch calculations. This paper presents voltage dependant EQE curves for both single junction and double junction a-Si solar cells, along with dispersion curves extracted from these data. In the case of single junction a-Si devices the mismatch factor is known to be voltage dependent and a similar trend is shown to apply to multi-junction devices as well. However, the error introduced into current voltage calibrations due to this bias dependence is found to be $<1 \%$ for spectral mismatch calculations.
\end{abstract}

\section{Introduction}

Multi-junction photovoltaic devices have the potential to provide higher efficiencies than single junctions and could therefore reduce the cost of solar electricity [1]. In the case of amorphous silicon (a-Si) devices, they could also reduce the impact of Staebler-Wronski degradation [2]. However, the characterization of multi-junction solar cells presents additional challenges over that of single junctions [3] and their indoor current-voltage $(\mathrm{I}-\mathrm{V})$ calibration is affected by greater uncertainties. Accurate $\mathrm{I}-\mathrm{V}$ calibration requires the $\mathrm{I}-\mathrm{V}$ measurements to be corrected with external quantum efficiency (EQE) measurements and spectral mismatch calculations. However, EQE is not independent of applied bias and so, in the context of carrying out measurements for use in energy rating standards, there is some debate on how to bias amorphous silicon solar cells to get an appropriate assessment of their quantum efficiency.

This paper aims to contribute to improvement of multi-junction device calibration by reporting the effects of bias on the EQE of a-Si single and double junction devices. It is shown that the bias voltage leads to small changes in the spectral mismatch correction factor. This might further complicate the accurate characterization of multi-junction devices as the mismatch factor may need to be calculated on a point by point basis. On the topic of multijunction characterization, a-Si double junction devices are particularly interesting as the junctions 'share' certain wavelengths. This is shown to result in a different behaviour than previously reported for III-V devices.

\section{Experimental methods}

The EQE measurement system used during this work is based on a dual-lamp light source and a series of narrow band-pass interference filters [4]. Measurement systems based on filters are optically more efficient than those based on grating monochromators and can therefore provide larger illumination areas. This allows complete illumination of devices, which minimises edge effects during EQE measurements. For this system, an area of $15 \times 15 \mathrm{~cm}^{2}$ is illuminated by the monochromatic illumination, allowing both the device under test and a calibrated reference device (c-Si photodiode) to be measured side-by-side. The monochromatic illumination was chopped at $175 \mathrm{~Hz}$ and the resulting photocurrents were measured simultaneously for both devices with lock-in amplifiers connected across $1 \Omega$ shunt resistors. A programmable four quadrant source meter was connected in series with the device under test to allow control of the device operating voltage during the EQE measurements. The system is flexible and can deal with all kinds of different technologies, as long as devices are within the specified size of illumination,. The size limitation is required because 
the system's calibration is based on overilluminating devices homogeneously as compared to spot measurements carried out more commonly.

The reference device used during this work was a crystalline Si photodiode quantitatively calibrated at ESTI. The optical design of the measurement system reduces the inhomogeneity of the monochromatic illumination to less than $\sim 10 \%$ across the illuminated area. However, since the EQE measurements presented here were not corrected for the precise irradiance distribution across the measurement area, they should be treated as qualitative.

Since multi-junction devices consist of two or more current sources in series, one of the junctions will normally be acting as the current limiting junction during operation. An accurate assessment of the $\mathrm{EQE}$ of the individual junctions requires that the junction under test be the current limiting junction in the stack. As described previously [3], this may be achieved by tuning the spectrum of the bias light used during measurement. Bias lighting was provided during this work by LED arrays driven from a high-stability programmable power supply. The bias lighting was able to provide sufficient irradiance for the a-Si devices under test to produce approximately $10 \%$ of their one-sun short circuit currents $\left(I_{s c}\right)$. The EQE set-up allowed I-V measurements to be made in-situ under the bias lighting. This permitted a spectrometric $[5,6]$ characterization of multi-junction devices to be performed prior to EQE measurements, allowing easy identification of the bias light conditions necessary to measure each sub-cell.

This paper reports the measurements of two types of amorphous silicon devices, single and double junction technologies, of $50 \mathrm{~mm} \times 50 \mathrm{~mm}$ of the same manufacturer. The single junction device is a single cell, the multi-junction has three cells in series.

\section{Results and discussion}

Initially, a single junction, single cell a-Si device was measured using the EQE system under fixed LED bias lighting and varying bias voltage conditions. The resulting set of EQE curves is shown in Figure 1, labelled with the voltage applied (in Volts). It can be seen that the EQE of the device decreases slowly for biases from $-1 \mathrm{~V}$ to $+0.5 \mathrm{~V}$ and then much more rapidly as the applied bias approaches then passes the maximum power point voltage $(0.58 \mathrm{~V}$ under the bias light level used for these measurements). The lower plot in Figure 1 shows the same data plotted on an expanded vertical axis in order to show clearly the behaviour of the device at high forward biases. At biases above approximately $+1.3 \mathrm{~V}$, the photocurrent is observed to reverse direction above a certain wavelength, leading to negative values of EQE. The transition wavelength is observed to decrease with the applied voltage. This effect of applied bias has been observed before for a-Si devices [7], though at much lower voltages with respect to the open circuit voltage $\left(V_{o c}\right)$. Numerical simulations of the carrier transport equations [8] show that this shift to higher voltages is consistent with a decreased i-layer thickness. It is noted that the change in direction of the photocurrent now occurs at such high forward biases as to be largely irrelevant for the normal operation of such devices.
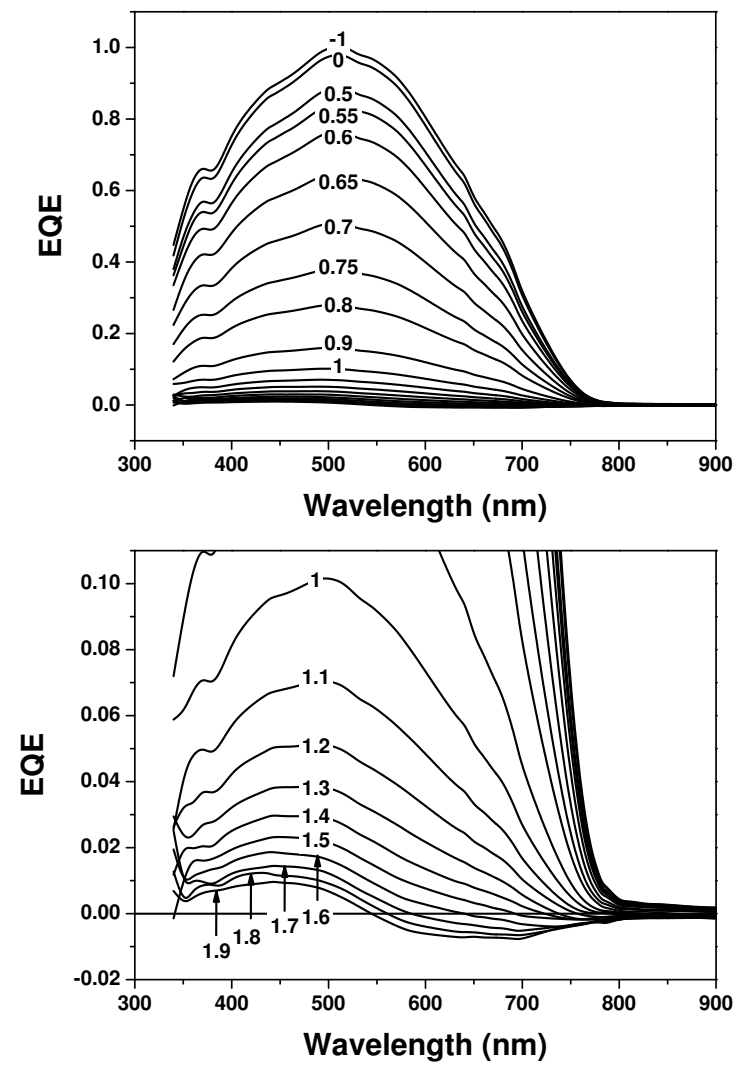

Figure 1: Top) Variation in EQE of an a-Si single junction, single cell device. Bottom) The same data, plotted on an expanded y-axis to highlight the behaviour at high forward bias. Labels indicate the bias voltage in Volts.

Comparison of the EQE curves measured at $-1 \mathrm{~V}$ and $0 \mathrm{~V}$ shows that the EQE of the device is higher 
at larger reverse biases. If the absolute EQE were used to correct the short circuit current of an I-V measurement of this device, using the $-1 \mathrm{~V}$ reverse biased EQE in place of the $0 \mathrm{~V}$ EQE would lead to a $3 \%$ overestimation of the $I_{s c}$. If the same two data sets were used to perform a spectral mismatch correction, the error in miss-match factor would be only $0.1 \%$ since it is the relative shape of the EQE that matters and not the absolute scaling. This effect is of particular importance when measuring multijunction devices as the limiting junction will operate at approximately the bias voltage applied to the device minus the $V_{o c}$ of the non-limiting junction [3].

Prior to measuring the EQE of tandem junction mini-modules, it was necessary to establish appropriate bias lighting conditions. This was achieved by performing a series of I-V measurements at fixed blue and varying amber LED intensity. The $V_{o c}$, fill factor $(F F)$ and $I_{s c}$ values extracted from one such set of I-V curves are shown in Figure 2. This method for finding the matching point of two junctions in a multi-junction device was previously presented for constant total irradiance as spectrometric characterization $[5,6]$. Here, the total irradiance is not kept constant and so the $I_{s c}$ and $V_{o c}$ values increase continuously as the intensity of the longer wavelength source is increased. However, the local minimum in $F F$ and the change in the rate of increase of $I_{s c}$ are still clearly visible. It was previously reported that a-Si tandem devices exhibit the $F F$ of the limiting junction [3], a phenomenon that was reported only for badly shunted III-V devices [6]. However, our measurements show that the a-Si devices measured during this work do exhibit the same local minimum in $F F$ as III-V devices, consistent with solution of the single-diode-model equations for two (nonshunted) series connected devices. The bias light conditions used for the measurements reported below were approximately 0.1 and 0.7 amber intensity, respectively, as defined by Figure 2 .

Both the top and bottom junctions of this two-cell, two-junction mini-module were then measured individually. The resulting sets of EQE curves are shown in the top and middle panes of Figure 3. The lower pane in Figure 3 shows the sum of the two sets of curves, together with the individual data sets and vertical lines indicating the peak emission wavelengths of the LED bias lights used to make these measurements. In order to measure the limiting junction under short circuit conditions, it would be necessary to apply approximately $1.3 \mathrm{~V}$ to the tandem device (i.e. approximately half of its $\left.V_{o c}\right)$. Comparison of the EQE curves measured at bias voltages up to $2 \mathrm{~V}$ therefore allows the overestimation of device $\mathrm{EQE}$ due to failure to apply sufficient forward bias to be seen clearly. The effect is evident for both junctions, though is more pronounced for the top junction.

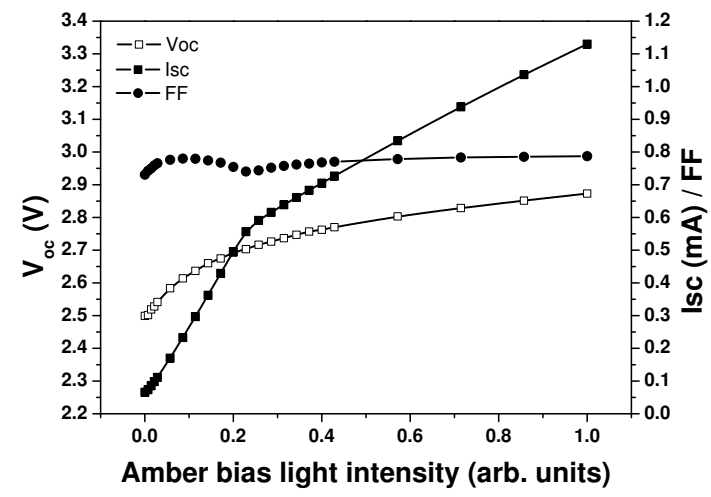

Figure 2: Cell parameters extracted from I-V curves measured from a tandem mini-module, with constant blue and varying amber bias light intensities. At the lowest levels of illumination, non-linear effects due to low irradiance conditions are visible, indicating the minimum illumination required for robust measurements.

Once a sufficiently large bias voltage is applied to push the operating point of the sub-cells past approximately their maximum power point voltages, neither junction is able to limit the current production. At this point a response is measured from both junctions during the EQE measurement and the summed EQE curves therefore do not represent the true behaviour of the tandem device. 

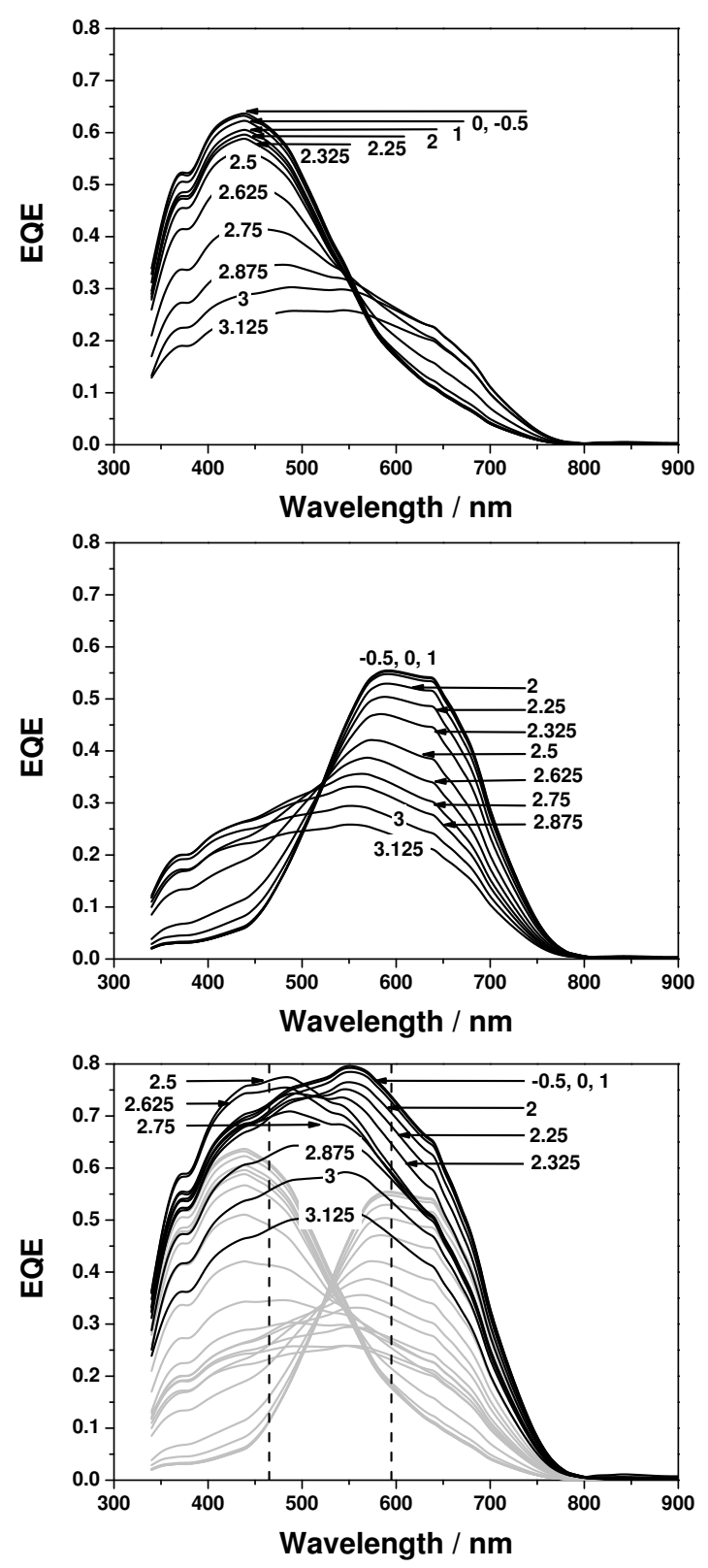

Figure 3: EQE curves measured from an a-Si two-junction, two-cell mini-module. Labels indicate the bias voltage in Volts. Top) Top junction limited. Middle) Bottom junction limited. Bottom) Summed data. The vertical lines indicate the peak emission wavelengths of the LED bias lights.

The quantity $q=-\operatorname{EQE}(\lambda, V) / \operatorname{EQE}(\lambda,-1 \mathrm{~V})$ has previously been defined as a useful quantity for investigating the dispersion of a-Si devices [7]. Figure 4 shows the quantity $q$ plotted for three wavelengths for the single junction and double junction devices detailed above. Both the $544 \mathrm{~nm}$ and $700 \mathrm{~nm}$ single junction curves cross the $q=0$ line as the bias voltage is increased. The maximum voltage applied was not sufficient to reverse the direction of the photocurrent at wavelengths as short as $405 \mathrm{~nm}$. This can also be due to internal mismatch between the cells, where this phenomenon will be more difficult to achieve. The variation in series resistance and the scribing resistances are expected to make a lower contribution. Although not clear from Figure 4, the $405 \mathrm{~nm}$ and $544 \mathrm{~nm}$ curves cross at approximately $0.85 V_{o c}$, due to the change in the relative shape of the EQE curves with applied bias. This change in shape will affect spectral mismatch corrections, even when the absolute spectral response values are ignored. However, the effects are small, calculated to be $<1 \%$ variation in missmatch factor for this device up to $1.5 \times V_{o c}$.

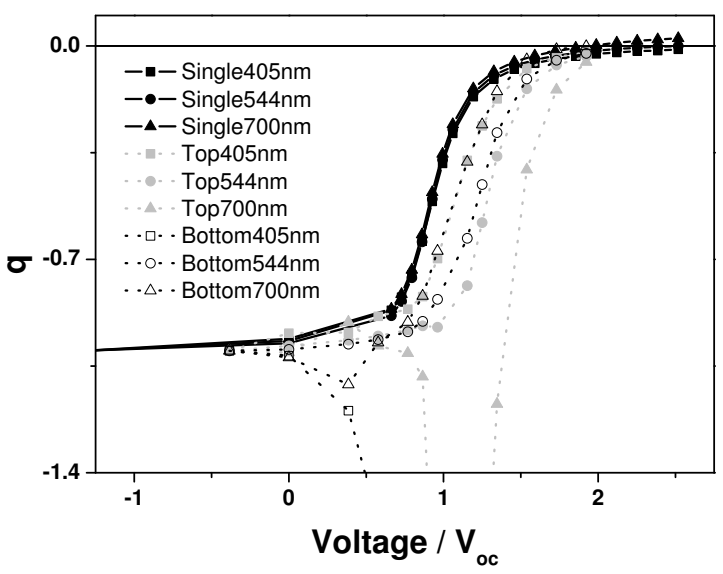

Figure 4: Dispersion curves for a single junction device and the individual junctions of a tandem device.

The $q$ values calculated for the individual junctions of the tandem device exhibit similar behaviour to the single junction device for the wavelengths to which they respond strongly. However, for the wavelength outside of their main response region (i.e. $700 \mathrm{~nm}$ for the top junction, $405 \mathrm{~nm}$ for the bottom junction), the value of $q$ becomes significantly more negative than -1 once the sub-cell being measured ceases to properly limit the current. This is due to the contribution of the other junction to the measured value of $\operatorname{EQE}(\lambda, V)$.

\section{Conclusions}

EQE measurements of both single and double junction a-Si devices have been reported and show that the bias voltage applied during device measurement has a strong effect on the measured response. For single junction devices, the forward bias required to reverse the direction of the 
photocurrent was found to be significantly higher than reported previously and numerical simulation showed that this is consistent with a reduction in the i-layer thickness. For multi-junction devices, the inability to keep one junction limiting the current while operating the device at voltages above its maximum power point voltage prevents full investigation of the dispersion of the EQE curve. This effect will prevent the calculation of a voltage dependent spectral miss-match factor for a full I-V measurement. However the calibration error due to this limitation is likely to be small as dispersion effects only become significant at large forward bias voltages.

Finally, the measurements presented here show the suitability of using LEDs as bias lighting to enable measurement of the individual junctions in multijunction PV devices.

\section{References}

[1] A. S. Brown and M. A. Green. Prog. in Photovoltaics: Res. and Applications 10 (2002) 299307.

[2] D. L. Staebler and C. R. Wronski. Applied Physics Letters 314 (1977) 292-294.

[3] J. Burdick and T. Glatfelter. Sol. Cells 18 (1986) 301-314.

[4] C. J. Hibberd, M. Bliss, H. M. Upadhyaya and R. Gottschalg. Proceedings of the Photovoltaic Science Applications and Technology Conference (2009) 239-242.

[5] R. Adelhelm and K. Buecher. Sol. Energy Mater. and Sol. Cells 50 (1998) 185-195.

[6] M. Meusel, R. Adelhelm, F. Dimroth, A. W. Bett and W. Warta. Prog. in Photovoltaics: Res. and Applications 104 (2002) 243-255.

[7] J. Bruns, S. Gall and H. G. Wagemann. J. of Non-Crystalline Solids 137-138 (1991) 1193-1196.

[8] C. Monokroussos, R. Gottschalg and A. N. Tiwari. Conference record of the twenty third European photovoltaic solar energy conference (2008). 